\title{
Lobectomy for Indeterminate Lung Tumors with a Strong Suspicion of Lung Cancer
}

\author{
Noritoshi Nishiyama, MD, PhD, Koshi Nagano, MD, Nobuhiro Izumi, MD, PhD, \\ Keiko Tei, MD, Shoji Hanada, MD, Hiroaki Komatsu, MD, and Shigefumi Suehiro, MD, PhD
}

\begin{abstract}
Wedge resection for tissue diagnosis of indeterminate lung tumors that is strongly suspected of being lung cancer, is sometimes difficult, and lobectomy, followed by a thorough pathological examination, is required. In the present report, four cases are presented, and the following indications, which have never been discussed before, are recommended for lobectomy without a pre-resectional diagnosis. First, where larger tumors are involved, and lobectomy is expected to result in a more favorable patient status and second, where the lesions are deeply located near major pulmonary vessels, or the patient is not a candidate for wedge resection or segmental resection. In each case, tolerance to surgery and detailed, informed consent for potentially complete resection are mandatory.
\end{abstract}

Keywords: lobectomy, lung cancer surgery, lung cancer, diagnosis

\section{Introduction}

For tissue diagnosis of an indeterminate lung tumor that is strongly suspected of being lung cancer, wedge resection is sometimes difficult because of tumor size or location. Intra-operative needle aspiration biopsy can be considered when tumor biopsy via flexible bronchoscopy (FB), or using transthoracic needle aspiration biopsy (TTNA), fails to prove malignancy in tumors with a high rate of false negatives. ${ }^{1-3)}$ There are numerous lesions where an easy wedge resection or TTNA cannot be carried out, and lobectomy followed by thorough pathological examination is required. In the present report, four cases with indeterminate lung tumors are presented, and the indications for lobectomy, which have never been reported before, are discussed.

$\overline{\text { Department Thoracic Surgery, Osaka City University Graduate }}$ School of Medicine, Osaka, Osaka, Japan

Received: May 9, 2011; Accepted: September 9, 2011

Corresponding author: Noritoshi Nishiyama, MD, PhD. Thoracic Surgery, Osaka City University Graduate School of Medicine, Osaka, Osaka 545-8585, Japan

Email: m1364552@msic.med.osaka-cu.ac.jp

(C)2011 The Editorial Committee of Annals of Thoracic and Cardiovascular Surgery. All rights reserved.

\section{Cases}

From April through September in 2010, among 35 patients who underwent lobectomy for primary lung cancer, four patients underwent lobectomy followed by thorough pathological examination without pre-resectional diagnosis.

Case 1. A 75-year-old man with a 35-mm pulmonary tumor located in the left lower lobe, detected by a screening chest X-ray and computed tomography (CT) (Fig. 1), was referred to our hospital. Positive emission tomography (PET) showed the accumulation of tracer with a $\mathrm{SUV}_{\max }$ of 2.9-3.5 in the tumor and serum carcinoembryonic antigen (CEA) was elevated to $13 \mathrm{ng} / \mathrm{ml}$ (normal range: $<5.0 \mathrm{ng} / \mathrm{ml}$ ).

Case 2. A 46-year-old man complaining of headache and dizziness underwent complete tumor resection of a 5 $\mathrm{cm}$ tumor in the left cerebellum. Pathological examination revealed that the tumor was metastatic carcinoma with an epithelial nature. A CT scan detected a 7-cm mass in the right upper lobe involving the chest wall (Fig. 2). PET revealed the accumulation of tracer with a $\mathrm{SUV}_{\max }$ of $4.5 \mathrm{in}$ the tumor, whereas serum tumor markers were within the normal range.

Case 3. A 70-year-old woman with stable adult T-cell 


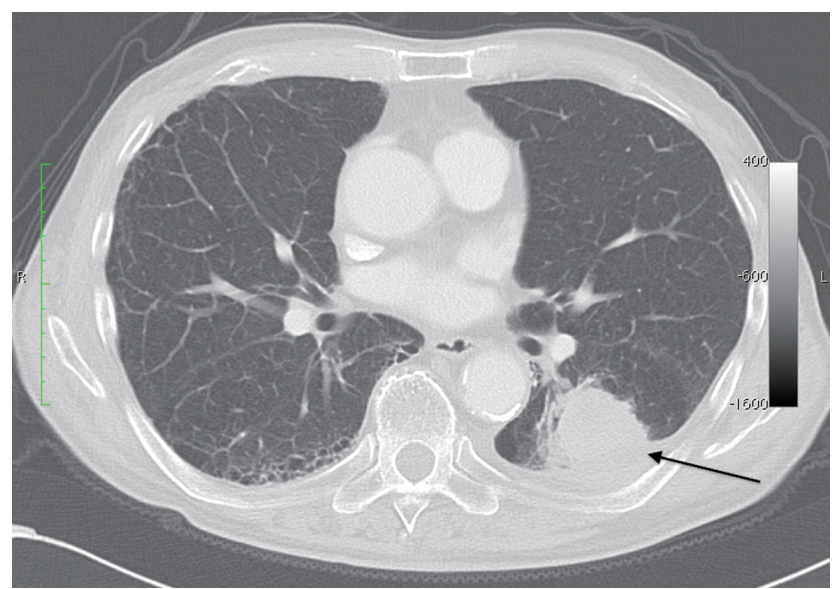

Fig. 1 Preoperative chest computed tomography showing a tumor measuring $35 \mathrm{~mm}$ in diameter in the left lower lobe (arrowed).

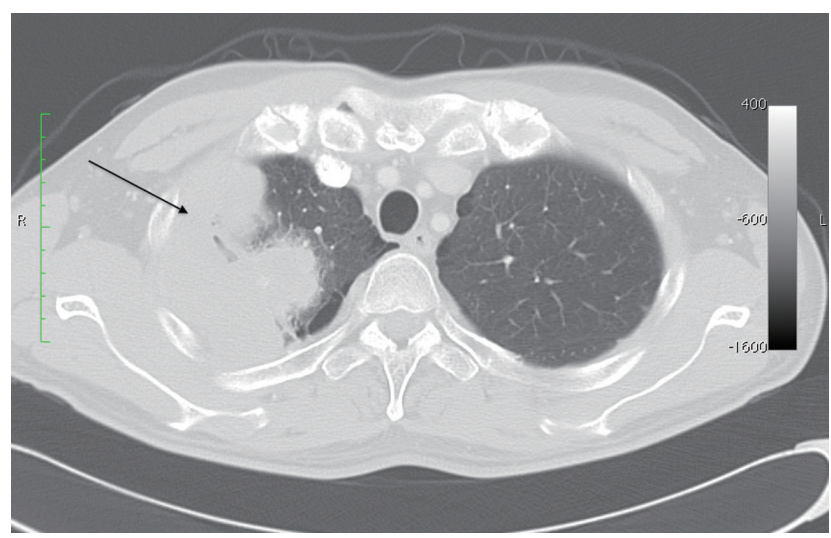

Fig. 2 Preoperative chest computed tomography showing a tumor measuring $35 \mathrm{~mm}$ in diameter in the right lower lobe (arrowed). Infiltrative shadow is clearly evident around the tumor. This has been followed up for 7 years.

leukemia/lymphoma (ATLL) and a $35 \mathrm{~mm}$ pulmonary tumor in the right lower lobe, detected by a chest CT for follow-up of an infiltrative shadow in the same lobe that had existed for 7 years (Fig. 3), was referred to our department. Although PET showed no accumulation of tracer in the tumor, serum CEA was elevated to $9 \mathrm{ng} / \mathrm{ml}$.

Case 4. A 75-year-old woman was referred to our hospital with a $20-\mathrm{mm}$ tumor detected by a screening chest X-ray and CT and located in the left upper lobe near the pulmonary artery, across the upper and lingular segments (Fig. 4). PET showed no accumulation of tracer in the tumor, and serum tumor markers were within the normal range.

In all four patients, although lung cancer was strongly suspected, transbronchial biopsy through FB failed to

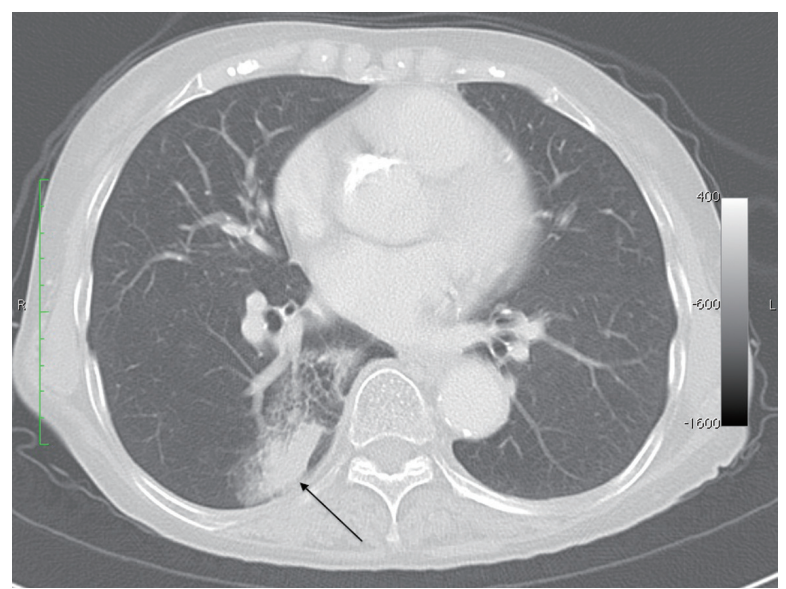

Fig. 3 Preoperative chest computed tomography showing a tumor involving the chest wall measuring $7 \mathrm{~cm}$ in diameter in the right upper lobe (arrowed).

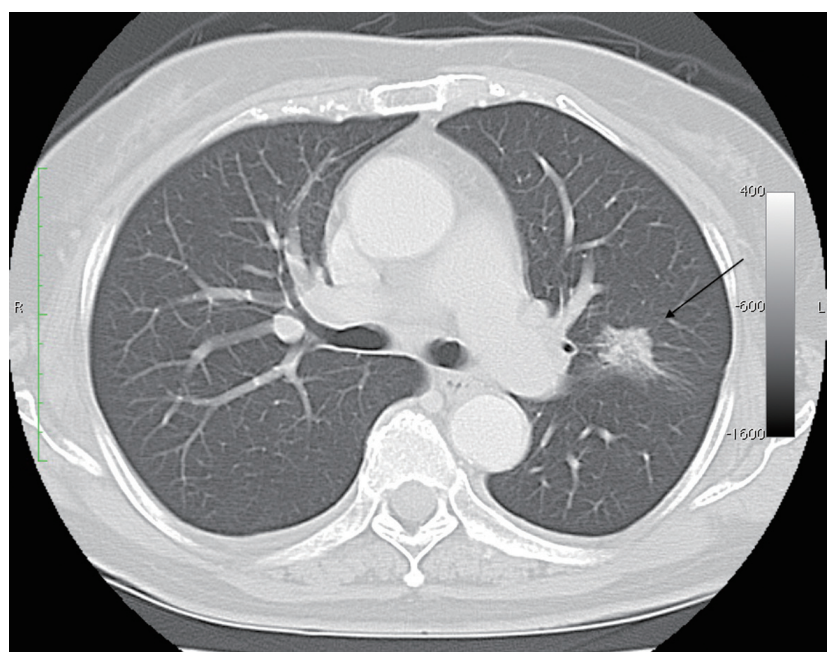

Fig. 4 Preoperative chest computed tomography showing a tumor with spiculation measuring $20 \mathrm{~mm}$ in diameter near the pulmonary artery across the upper and lingular segments in the left upper lobe (arrowed).

prove malignancy. The patients were recommended surgery without TTNA, subsequent to detailed consultation and informed consent for potentially complete resection, including risks of lobectomy for definitively benign disease resulting in over-resection. Prior to surgery, cardiopulmonary assessment, including spirometry, electrocardiogram, ultracardiography or coronary angiography as necessary, was performed, and all patients were estimated as tolerable to lobectomy. Surgical approaches were posterolateral thoracotomy for Case 1 and 2, in case of chest wall resection, and video-assisted thoracic surgery with a mini-axillary thoracotomy and two ports for Case 3 and 4 (Table 1). 
Table 1 Preoperative cardiopulmonary assessment and surgical approach

\begin{tabular}{|c|c|c|c|c|c|c|c|}
\hline & $\begin{array}{l}\text { VC (litters) } \\
(\% \text { prediced })\end{array}$ & $\begin{array}{l}\text { FVC (litters) } \\
(\% \text { prediced })\end{array}$ & $\begin{array}{c}\text { FEV1.0 (litters) } \\
\text { (\% prediced) }\end{array}$ & $\begin{array}{l}\text { FEV1.0 } \\
(\% \text { FVC) }\end{array}$ & $\begin{array}{c}\text { DLco } \\
(\% \text { predicted })\end{array}$ & $\begin{array}{c}\text { cardiac } \\
\text { screening }\end{array}$ & $\begin{array}{l}\text { surgical } \\
\text { approach }\end{array}$ \\
\hline Case 1 & 3.63 (115) & 3.63 (115) & 2.02 (112) & 56 & 81 & $\begin{array}{l}\text { ECG, UCG, } \\
\text { CAG for OMI }\end{array}$ & PL \\
\hline Case 2 & $5.55(140)$ & $5.41(137)$ & 4.12 (154) & 76 & 80 & ECG & PL \\
\hline Case 3 & $1.91(88)$ & $1.83(84)$ & $1.12(75)$ & 61 & 80 & ECG, UCG & $\begin{array}{l}\text { VATS with mini-axillary } \\
\text { thoracotomy }\end{array}$ \\
\hline Case 4 & $2.06(95)$ & $2.06(95)$ & $1.51(104)$ & 73 & 144 & ECG, UCG & $\begin{array}{l}\text { VATS with mini-axillary } \\
\text { thoracotomy }\end{array}$ \\
\hline
\end{tabular}

VC: vital capacity: FVC: forced vital capacity; FEV1.0: forced expiratory volume in one second; DLco: diffusion capacity of lung carbon monoxide; UCG: ultracardiography; CAG: coronary angiography; OMI: old myocardial infarction; PL: posterolateral thoracotomy; VATS: video-assisted thoracic surgery

During surgery, intra-operative incisional biopsy or needle biopsy were avoided because of the possibility of a false negative result, or the inability to achieve a deep punction, due to the difficult location of the tumor. In each case, lobectomy (in Case 2 lobectomy was combined with resection of the affected chest wall) was performed and followed by immediate intra-operative pathological examination of the tumor. The diagnosis was lung cancer, and additional mediastinal lymph node dissection was performed. The definitive diagnoses in Cases 1,2, 3, and 4 were moderately differentiated squamous cell carcinoma pT2aN2M1b (chest wall), pleomorphic carcinoma pT4 (intrapulmonary metastasis in the right lower lobe) N1M1b (brain), bronchioloalveolar carcinoma pT3NOM0 (including the infiltrative shadow around the tumor), and moderately differentiated papillary adenocarcinoma pT1bNOM0, respectively ${ }^{4}$. Each patient underwent an uneventful postoperative course and was discharged with a plan for adequate postoperative treatment.

\section{Discussion}

Since the prevalence of indeterminate lung tumor in the general population is unknown, Swenson and colleagues ${ }^{5)}$ reviewed 629 patients with indeterminate solitary pulmonary nodules. They identified three clinical factors (age $>40$ years, positive smoking history and history of other cancer) and three radiologic characteristics (tumor over $2 \mathrm{~cm}$ in diameter, spiculation present and location in the upper lobe) that were independent predictors of malignancy, also found that $12 \%$ of the patients remained indeterminate. ${ }^{5)}$ In the diagnosis of indeterminate lung tumors that are strongly suspected of being cancerous, the invasiveness of tissue sampling ranges from thoracentesis to classic thoracotomy. ${ }^{1)}$ For peripheral lesions, the false negative rate of FB or TTNA is high and it is generally not useful in ruling out cancer, making reliance on a negative result untenable. ${ }^{2,3)}$

The next step is surgery and some authors have advocated immediate resection of all indeterminate solitary pulmonary nodules. ${ }^{2,6,7)}$ When a peripheral tumor is safely and entirely removed using wedge resection, a correct diagnosis will be obtained. However, there exist quite a few lesions where an easy wedge resection cannot be undertaken. In addition, similar to the high rate of false negative results associated with the use of TTNA, intraoperative incisional or needle biopsy can result in a false negative pathological diagnosis. In addition, we had experienced a case of a large tumor in the right upper lobe, diagnosed as large cell carcinoma with the lobectomy specimen, nevertheless an intraoperative tumor biopsy was screlosing hemangioma. Since intraoperative biopsy is mandatory from an oncological point of view, surgeons have to be aware in practical settings that, to avoid loosing a chance of complete resection, a false negative result cannot exclude the need for a lobectomy. Therefore, in certain circumstances, lung tumors with a strong suspicion of lung cancer should be resected by lobectomy, and followed by a thorough pathological examination, without a pre-resectional diagnosis. One of these conditions is when lobectomy is expected to result in a more favorable patient status, regardless of definitive diagnosis where larger tumor volume is involved. In this report, Case 2 corresponds to this criterion and we believe even a noncurative lobectomy because of brain metastasis led the patient to at least macroscopic tumor-free condition, which contributed to his better status for postoperative adjuvant therapy. A second condition is when the lesion is deeply located near the major pulmonary vessels, or is not a candidate for wedge resection or segmental resection. 
Tumors in Cases 1 and 3 were obviously too large for wedge resection or segmental resection, and the tumor in Case 4 located near the major pulmonary artery across the segments. The following conditions are common to each case: complete resection should be performed, detailed informed consent is required including risks of lobectomy for definitively benign disease resulting in over-resection, and the patient should have good tolerance to surgery.

Increased serum tumor markers can provide useful information. PET is now widely accepted for detecting malignancy; it has an average sensitivity and specificity of 0.97 and 0.78 , reported in a meta-analysis evaluation. ${ }^{8)}$ Thus, under such conditions, failure of diagnosis with FB can be followed by surgery involving complete tumor resection, without TTNA or intraoperative biopsy.

\section{Conclusion}

For an indeterminate lung tumor that is strongly suspected of being lung cancer, lobectomy, followed by a thorough pathological examination is required, without a pre-resectional diagnosis. First, where larger tumors are involved, and lobectomy is expected to result in more favorable patient status and second, where the lesions are deeply located near major pulmonary vessels, or is not a candidate for wedge resection or segmental resection. In each case, tolerance to surgery and detailed, informed consent for potentially curative resection are mandatory.

\section{References}

1) Savage C, Morrison RJ, Zwischenberger JB. Bronchoscopic diagnosis and staging of lung cancer. Chest Surg Clin N Am 2001; 11: 701-21, vii-viii.

2) Midthun DE, Swensen SJ, Jett JR. Clinical strategies for solitary pulmonary nodule. Annu Rev Med 1992; 43: 195-208.

3) Rivera MP, Mehta AC. Initial diagnosis of lung cancer. ACCP evidence-based clinical practice guidelines. 2nd ed. Chest 2007; 132: 1315-485.

4) Sobin LH, Gospodarowicz MK, Wittekind CH, eds. UICC (International Union Against Cancer) TNM classification of malignant tumors. 7th ed. New York: Wiley-Blackwell, 2009.

5) Swensen SJ, Silverstein MD, Ilstrup DM, et al. The probability of malignancy in solitary pulmonary nodules. Application to small radiologically indeterminate nodules. Arch Intern Med 1997; 157: 849-55.

6) Higgins GA, Shields TW, Keehn RJ. The solitary pulmonary nodule. Ten-year follow-up of veterans administration-armed forces cooperative study. Arch Surg 1975; 110: 570-5.

7) Ray JF 3rd, Lawton BR, Magnin GE, et al. The coin lesion story: update 1976. Twenty years' experience with thoracotomy for 179 suspected malignant coin lesions. Chest 1976; 70: 332-6.

8) Gould MK, Maclean CC, Kuschner WG, et al. Accuracy of positron emission tomography for diagnosis of pulmonary nodules and mass lesions: a meta-analysis. JAMA 2001; 285: 914-24. 